\title{
Constraints on computational models of auditory scene analysis, as derived from human perception
}

\author{
Albert S. Bregman \\ Psychology Department, McGill University, \\ 1205 Docteur Penfield Ave., Montreal, Quebec, Canada
}

\begin{abstract}
Auditory scene analysis (ASA) groups the sensory evidence derived from a mixture of sound sources and derives separate descriptions of the individual sources. There are two possible ways of doing this: (a) using knowledge of the properties of certain classes of sounds, such as speech, in a top-down fashion; (b) using, in a bottom-up manner, those properties in the input (such as harmonic relations) that are typically found when two or more sound sources (e.g., voice and footsteps) affect the input at the same time. Some properties of the ASA done by humans are discussed and proposed as specifications for a computational model: the adding up of evidence, the coherence of the ASA system, the combining of top-down and bottom-up constraints, as illustrated in the duplex perception of speech, and the propagation of influences across the auditory field.
\end{abstract}

Keywords: Hearing, Scene analysis, Speech perception, Auditory perception

PACS number: 43. 66. Ba

The "scene analysis problem" is the name given to the difficulty faced by perceptual systems in using the array of sense data to build descriptions of distinct objects and events. In audition, the difficulty is that the acoustic energy that has come from many concurrent and interleaved events is mixed together at the ear. There is only a single waveform. Yet the auditory system must use it to perceive a number of separate sounds. We know that peripheral mechanisms in the auditory system start by laying out the incoming spectrum along a frequency dimension in the inner ear. Taken over time, the information in this form would roughly resemble what we obtain in a spectrogram.

If the many sounds active at the same time occupied different regions of frequency or never overlapped in time, auditory scene analysis (ASA) could separate them by their positions in this neural spectrogram. However, sounds are overlapped in time and frequency. So any active part of the spectrum could contain the energy from two or more sources, and activity in widely spaced parts of the spectrum might come from a single source. Yet unless the system sorted out the spectrum correctly, it might not derive the correct properties for concurrent sounds, such as voices. Not only simultaneous acoustic energy but also energy distributed over time must connected appropriately. Getting the sequential connection wrong might cause the listener to hear a false message formed from a concatenation of syllables of two different speakers.

ASA makes use of two sources of information: (a) regularities in the incoming acoustic array that serve as evidence for the number of environmental events ("bottom-up" information), and (b) familiarity with particular types of sounds such as voices, instruments, and machines ("top-down" information). I believe that the processes that use the bottom-up acoustic regularities are primitive, i.e., unlearned, and that the regularities they exploit hold true across broad classes of sounds.

My own research has focused on these primitive processes, which take advantage of the fact that regularities in the sound reflect regularities in the environment. Here is an example: Many vibrating objects, including the human vocal cords, emit a set of frequencies (harmonics) that are multiples of the fundamental. Therefore the presence, in the sense 
data, of a set of frequencies related to a common fundamental is a symptom of the presence of a single sound which has emitted all these harmonics. To give another example, the events in the world do not change their spatial locations rapidly, or if they do, they tend to move continuously from one position to another. Therefore if we hear a rapid alternation of sounds coming from two distinct locations, with no sounds between them, it is a good bet that we are hearing two sound sources, not one. We should group the sounds sequentially within locations, not across. Similarly, natural sounds tend not to abruptly change in pitch or in spectral region. If the sense data registers such discontinuities, we can conclude that the sound is coming from more than one environmental event.

These regularities are only true with a certain probability. Not all sounds are harmonic and occasionally the sound coming from a certain event can change its properties abruptly in the middle. However, if the auditory system looks for, and uses, a large number of probably-valid regularities at the same time, adding up the advice that they give, it has a good chance of grouping the sense data in the right way.

I would like to address the remainder of this preface to two issues. The first is whether bottom-up ASA is a coherent system and the second is how it is related to top-down processes of recognition, especially speech recognition.

The first question concerns the coherence of the system that performs primitive ASA. This is of most interest to researchers studying the revelant brain processes. One could imagine two opposing theories about how ASA was achieved: (1) There could be a system of interconnected processes, all evolved to serve ASA, close to one another in neural location. (2) There could be a number of processes, scattered across different levels of the auditory system whose only similarity was that they happened to contribute to solving the ASA problem, each also making contributions to perceptual processes unrelated to ASA. For example, it is possible that the activity of the pitch detection system happens to scene-analysis goal by grouping harmonics, but this is incidental to the quite different role that it plays by assigning a pitch that can be used in pattern recognition.

Do we have any evidence that can decide among these two theories? One thing we know is that the various cues to the organization of the incoming signal always compete and collaborate. For example, if the difference in fundamentals is not quite enough to segregate a pair of alternating sounds, adding a difference in spectral peaks will cause segregation to occur. This collaboration makes it hard to maintain that the grouping of sequences of sounds into streams based on their fundamental frequencies actually takes place at one neural location, and that the actual grouping based on their spectral peaks takes place at another. To collaborate, information about the fundamental and the spectral peaks must reach some common neural region in which the final organization takes place. Different structures, perhaps at quite different neural locations, may extract the information upon which ASA is based. However, extracting it and using it to create an organization are two different things.

The second question is about the relation between bottom-up and top-down processes in auditory perception. Most of the findings in ASA have been about bottom-up cues. Examples are that the sequential segregation of sounds into different streams is favored when the sounds change abruptly in fundamental frequency, spectral range, spectral balance, or spatial location. Similarly the segregation of simultaneous sounds into separate streams is favored if they are far apart in frequency or spatial location, if they start and stop asynchronously, if their amplitude changes are independent, or if they consist of harmonics of different fundamentals. However, if we are to use this knowledge to understand the recognition of speech or other sounds, we must study how bottom-up processes interact with top-down ones.

The issue of down-up interaction is of central importance in computer-based models of recognition. There is a temptation, in such modeling, to arrange the interaction between recognition and primitive ASA without changing existing models of recognition. For example, a modeler of speech recognition might use the bottom-up processes to partition the spectral energy into separate signals that might have come from separate sources, and feed these to an speech recognizer. However, there is evidence that the human brain does not completely separate sounds in this way. While researchers are free to develop models as they wish, they should know at what point they have crossed the boundary between the modeling of humans and the building 


\section{A. S. BREGMAN: COMPUTATIONAL MODELS OF AUDITORY SCENE ANALYSIS}

of arbitrary systems of artificial intelligence.

Evidence about the interaction between bottom-up and top-down processes in humans comes from research on the perception of synthetic speech. An example is the phenomenon of "duplex perception." The listener is presented with speech syllables that can be distinguished from one another by a single short formant transition (for example "ga" versus "da"). Most of the syllable is presented to one ear, say the left, while the distinctive formant transition is presented to the other. Hearing this, the listener clearly perceives two sounds, one at each ear. This division of the incoming data into two streams is clearly the result of bottom-up processes. If the human top-down recognition processes were capable of accessing only one stream at a time, they would be unable to choose between "da" and "ga," since the evidence would have been separated into two streams. However, the listener does use the evidence from both ears to identify the syllable, despite the fact that two streams have been heard. This implies that top-down processes have access to more than one stream at a time.

However, it is not the case that speech recognition is simply a special system that is oblivious to the segregation performed by bottom-up processes. For example, it is easier to understand a talker when a competing voice is speaking in a different pitch range or at a different spatial location, or if the two voices are filtered in such a way that their spectra are distinct. These are factors known to affect ASA. Therefore we must look for something more complex than that either a total domination of topdown speech recognition processes by bottom-up ones or a total independence of speech recognition from bottom-up influences.

It is more reasonable to propose that bottom-up ASA processes operate by laying constraints on the selection of data by recognition processes, but the constraints are not absolute. One way to organize such a process in a model would be to have the bottom-up process assign a common "label" to acoustic elements presumed to come from the same source. This label could also contain a numerical value indicating the strength of the evidence favoring this grouping. Then when different schemas (say the ones for "da" and "ga") were searching for acoustic evidence, they would have to pay a penalty if they wished to incorporate elements that had different labels on them. The penalty would be proportional to the strength value assigned to each element. This arrangement would allow the duplex perception described earlier. Both the "da" and the "ga" recognizers would have to pay the same penalty for using data from two different ears. Therefore the choice between hearing the two syllables would not depend on the two-ear presentation but only on which syllable was best supported by the data. The idea of labeling would also explain why it was casier to segregate one talker from another one when bottom-up processes had labeled the data as belonging to two subsets. This added constraint could help avoid mixing the information from the two talkers.

Notice that this arrangement of processes would involve a modification of the structure of current speech recognizers, not just of the data which they receive, since they would have to accept sets of constraints. I am sure there is a reluctance to change the structure of existing speech recognition programs. It is easier to construct separate waveforms, and then feed these into already existing recognition programs. While doing this may be useful as a quick check on the effectiveness of the bottom-up processes, it deviates from what we know about recognition in humans.

There now exist formalisms, for example network models, that make it easier to add up a number of soft constraints on the final output. I am not referring to completely self-organizing networks, which seem to me to have a number of limitations, but to ones that have been carefully designed to have desirable properties. The required properties are not trivial to achieve. They include: (a) the ability to represent the grouping of sense data; (b) the ability to rapidly use the factors, known to affect primitive organization, to group information as it comes in, so that the grouping can play a role in the recognition; (c) the ability to added up constraints derived from either bottom-up primitive organizing processes or from top-down recognition networks; and (d) the ability to choose the "best" conceptual description of the input and to inhibit the competing ones. I am sure we will soon see interesting results using this class of model.

The two problems that I have discussed, the coherence of the system and the relation between primitive and knowledge-governed processes, are not the only ones that we face in understanding ASA. Many others remain: Does the grouping of data in 
one part of the frequency-by-time auditory field propagate influences that affect the grouping of other parts? How can we describe the more naturalistic sounds of everyday life in ways that tell us how they will be organized? These and many other difficult questions will provide the current generation of auditory researchers with exciting challenges for many years to come.

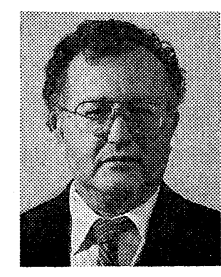

\begin{abstract}
Albert Bregman Albert Bregman is a professor of psychology at McGill University in Montreal, Canada. Born in Toronto, Canada (1936), his education includes a B.A. and M.A. from the University of Toronto, and a Ph.D. in Psychology from Yale (1963). He was an instructor in the psychology
\end{abstract}

department and a researcher at the Center for Cognitive Studies at Harvard (1963-65). He came to McGill in 1965 where he rose to the rank of Full Professor (1976). He has taught cognition, auditory perception, psychological theory, and experimental methods, and has developed a computer-based laboratory for the analysis and synthesis of sound and for the psychoacoustic testing of human subjects. Sabbaticals have taken him to Cornell, Sussex, and Stanford Universities. In his more than fifty publications on auditory scene analysis (ASA), he has studied the cues for, and the perceptual results of, ASA as well as its role in the perception of speech and music. In 1984 he received a Killam Research Fellowship from the Canada Council. This freed him from teaching to present his ideas in the form of a book, Auditory Scene Analysis, which was published by the MIT Press in 1990. 